Original Research Paper

\title{
Factors Affecting Radiographers in the Clinical Supervision of Radiography Students in a Limited Resource Setting: A Survey in Zambia
}

\begin{abstract}
Bwanga, O. ${ }^{1}$, Sichone, J.M. ${ }^{2}$, Kaonga, $P .^{3}$
Abstract

Background and Purpose: Clinical teaching and learning are an important aspect of radiography programmes. It enables radiography students to acquire knowledge, attitudes, skills, and competence under the clinical supervision of designated radiographers. However, there is a paucity of research on radiography clinical training and the factors that impact on it. This study aimed to evaluate factors that affect supervising radiographers in the clinical supervision of radiography students in Zambia.
\end{abstract}

Methods: A cross-sectional design was utilised in this study. A self-administered questionnaire was used to collect data from supervising radiographers $(\mathrm{N}=120)$ working in public hospitals affiliated with the schools of radiography and located in the Lusaka and Copperbelt provinces of Zambia. Data were analysed using descriptive and inferential statistical methods.

Results: The response rate was $75 \%(\mathrm{~N}=120 / 160)$. Supervising radiographers rated the current system of clinical supervision as being unsatisfactory. Factors that facilitated the clinical supervision process were an adequate supply of X-ray films, students sharing their learning problems and radiology department management support. On the other hand, factors that inhibited the clinical supervision process were a lack of guidelines, lack of theory amongst students, combined supervision of diploma and degree students, student overcrowding, lack of educational audits, unjustified imaging requests, insufficient clinical teaching knowledge, and inadequate faculty and hospital management supports.

Conclusion: Supervising radiographers encounter both facilitating and inhibiting factors in the facilitation of practice-based learning for radiography students. More collaboration amongst key stakeholders and supports are necessary to overcome the challenges identified in this study.

Keywords: Clinical supervision, facilitating factor, inhibiting factor, radiography student, supervising radiographer, Zambia

\section{Background}

Clinical supervision is a process by which a student and an experienced practitioner work together to improve the student's competencies

\footnotetext{
1. Midlands University Hospital Tullamore,

Radiology Department, Co. Offaly, Ireland

${ }^{2}$ University of Zambia, School of Health Sciences, Radiography Section, Lusaka, Zambia

3 University of Zambia, School of Public Health, Department of Epidemiology and Biostatistics, Lusaka, Zambia.

Corresponding author: Dr. Osward Bwanga (PhD) o.bwanga@yahoo.com
}

(University Ottawa, 2011).Initially, the training of X-ray assistants (the precursors to radiographers) was based on the apprenticeship model (Thomas \& Banerjee, 2013; Sutton, 2013). This model of training lacked the scientific underpinning of a formal education and the quality of the training varied according to the trainer's capacity and conscientiousness (University Ottawa, 2011; Sutton, 2013). For this reason, the College of Radiographers in the United Kingdom (UK) was set up in 1920 to formalise and improve the quality of radiography training (Thomas \& Banerjee, 2013). This is now a global trend.

DOI: http://doi.org/10.4038/seajme.v15i2.357

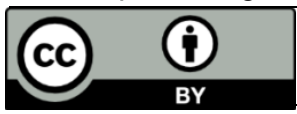


Effective clinical supervision of radiography students is important in many aspects. From an educational perspective, it allows students to safely integrate the knowledge gained in the classroom with practical experience (Baker \& Latham, 2013). From a supervising radiographer's perspective, it allows them to develop teaching, management, and communication skills (Baker \& Latham, 2013; Thompson \& Taylor, 2020). From the placement provider's point of view, it results in the provision of a quality healthcare delivery system. Msiska et al., (2014) argue that unsupervised students' experiences can lead to the acceptance of lower standards of care because students may not learn correct practices. From the patients' perspective, it has a positive effect on their safety and health outcome (Msiska et al., 2014). Lastly, from a regulatory and professional body's point of view, supervision ensures that patients are protected from any risks associated with students participating in healthcare delivery (Health Professions Council of Zambia, 2014; Radiological Society of Zambia, 2018).

In Zambia, there are currently three undergraduate radiography programmes: a three-year diploma in diagnostic radiography by Evelyn Hone College (EHC), a four-year Bachelor of Science in diagnostic radiography by the Lusaka Apex Medical University (LAMU) and a five-year Bachelor of Science in radiography by the University of Zambia (UNZA). The curricula of all three training programmes consist of theory and clinical practice. First-year students are exclusively involved in preclinical subjects such as anatomy and physiology, physics, and mathematics, as well as simulation of the clinical experience (LAMU, 2011; UNZA, 2017; TEVETA, 2018). In the second to final years, students continue with theory in radiography related subjects such as imaging techniques, equipment and pattern recognition, whilst simultaneously being attached to hospitals for clinical practice. During clinical practice, students are supervised by radiographers with more than two years of working experience in radiography. The supervising radiographers are responsible for reviewing the radiology request forms to determine the justification and the student's ability to perform the examination, assessing the condition of the patient and the complexity of the requested imaging examination whilst considering the student's level of competence, as well as reviewing and approving the completed images.

A review of the literature found a paucity of published information on radiography clinical training and the factors that impact on it. Anecdotal information pointed to complaints about the quality of clinical training for radiography students in Zambia. The objective of the study was, therefore, to evaluate factors that affect supervising radiographers in the clinical supervision of radiography students in Zambia. In this study, a radiographer means a healthcare professional with a diploma (radiography technologist) or a degree in diagnostic radiography.

\section{Methods}

\section{Study design and setting}

The study utilised a cross-sectional design. It was part of a larger study focusing on developing the strategies to support radiographers in the clinical supervision of radiography students in Zambia. The study setting was public hospitals affiliated with the schools of radiography and located in the Lusaka and Copperbelt provinces of Zambia. This setting, therefore, provided an ideal environment for conducting this study because most of the radiographer population is also based in these two provinces.

\section{Population and sampling}

There was a total of 180 radiographers working in thirteen study sites. Due to the small number of radiographers working in the two selected provinces, the entire population of interest was included. The inclusion of the entire population, therefore, yielded representative results (Bryman, 2016).

\section{Data collection}

Data were collected using a self-administered questionnaire which was developed based on 
the qualitative findings of a larger mixedmethods study. It was divided into two sections: demographic data of respondents and factors affecting supervising radiographers in the clinical supervision of radiography students. The first part of the questionnaire consisted of six questions on respondents' gender, age, education, position or rank, experience, and work location. The second part contained fifteen statements with a Likert scale of 1 to 5 .

After gaining ethics approval, a pilot study was conducted in October 2018 using a sample of five supervising radiographers to test the drafted questionnaire. The reliability analysis yielded a Cronbach Alpha value of 0.76 which was acceptable (Bryman, 2016). After the pilot study, questionnaires were administered to all 160 accessible population between October and December 2018, using postal and hand delivery.

\section{Data management and analysis}

Data were generally tested for normality using the Shapiro-Wilk test. Proportions and measures of central tendency were used to provide the descriptions of the data. In each category, comparisons were made using Kruskal-Walis and post hoc test (Dunn's Multiple Comparison Test) with significance set at 0.05 . Using a Wilcoxon signed-rank test with a hypothetical value of 60 , a comparison was made between the aggregate rating and the required mark for accreditation. The value of 60 percent is used as the Technical Education Vocational and Entrepreneurship Training Authority (TEVETA) score requirement for accreditation as an intermediate ranked institution offering training in Zambia (TEVETA, 2012)

\section{Ethical considerations}

The study was approved by the University of South Africa (UNISA) and Tropical Diseases Research Centre (TDRC) research ethics committees and permission sought from the Ministry of Health of Zambia. An information sheet was attached to each questionnaire explaining the aim and details of the study. The completion and return of the questionnaire confirmed respondents' consent to taking part in the study.

\section{Results}

\section{Demographic characteristics of respondents}

The respondents were drawn from two provinces (Lusaka and Copperbelt) of Zambia. The study had more males than females with the majority at the level of radiography technologist. A total of 120 supervising radiographers took part in the survey. Table 1 depicts the specific demographics of the respondents.

Table 1: Demographic characteristics of respondents

\begin{tabular}{cccc}
\hline Characteristic & Category & Proportion & Percentage (\%) \\
\hline \multirow{2}{*}{ Gender } & Male & 67 & 55.8 \\
& Female & 53 & 44.2 \\
\hline \multirow{4}{*}{ Position } & Radiography technologist & 81 & 66.7 \\
& Radiographer & 27 & 22.5 \\
& Senior radiographer & 5 & 4.2 \\
& Principal radiographer & 2 & 1.7 \\
& Chief radiographer & 5 & 4.2 \\
\hline \multirow{2}{*}{ Province } & Lusaka & 75 & 62.5 \\
& Copperbelt & 45 & 37.5 \\
\hline \multirow{2}{*}{ Education } & Diploma & 81 & 67.5 \\
& Bachelors & 33 & 27.5 \\
& Masters & 6 & 5.0 \\
\hline Age & & Median & IQR \\
\hline Work Experience & & 34 & $27-37$ \\
\hline
\end{tabular}


Factors related to the managerial component of the clinical supervision of radiography students

In the evaluation of issues that impact on the managerial component of clinical supervision of students, 5 factors (X-ray film availability, guidelines availability, overcrowding by students, combined supervision of diploma and degree students, and performance of audits) were investigated. Figure $1 \mathrm{~A}$ shows the box and whisker plot with associated comparisons. Concerning X-ray film availability, respondents' median rating of availability was 4 (IQR 2-4) with more than half $[\mathrm{N}=75(62.5 \%)]$ selecting 4 and 5 . Regarding the number of radiography students, more than half [ $\mathrm{N}=65(54.1 \%)]$ of the respondents believed that the radiology departments become overcrowded with students during clinical training, and hence rated this category as low with the median at 2 (IQR 2-4). Many [ $\mathrm{N}=49$ (40.8\%)] of the respondents, also indicated that they find it challenging to supervise diploma and degree radiography students in the same group, therefore rated the statement with the median at 3 (IQR 2-4). In another statement, respondents were asked whether educational audits of radiology departments affiliated with the schools of radiography are conducted periodically and the majority [ $\mathrm{N}=74(61.7 \%)]$ disagreed or strongly disagreed with the statement, hence giving it a low rating with the median rating at 2 (IQR 2-3). Lastly, the majority [ $\mathrm{N}=95(78.1 \%)]$ of the respondents believed that there is a lack of clinical supervision guidelines and this was the lowest ranked amongst the managerial factors. Overall, there was a significant difference in the rating across the five categories $(p<0.001)$ with a post $\mathrm{HOC}$ test showing the difference to be predominately arising from a high rating of availability of $X$-ray films $(p=0.001)$.

Factors related to the educational component of the clinical supervision of radiography students

Figure 1B below illustrates the rating of educational factors. In rating the radiography students' required knowledge before clinical training, the majority $[\mathrm{N}=98(81.7 \%)]$ of the respondents rated at 2 or less with the median at 2 (IQR 1-2). Respondents were asked whether the radiology department had an adequate written policy statement on professionalism, and more than half $[\mathrm{N}=72$ $(60 \%)]$ of the respondents rated this as 3 or less. Majority of respondents [ $\mathrm{N}=93(77.5 \%)$ ] indicated that the level of justified imaging requests was at 2 or less. This was similar for knowledge about clinical teaching and learning statement, where the majority [ $\mathrm{N}=84(71.7 \%)]$ of the respondents, believed that they lack the knowledge and rated this at 2 or less. The overall level of agreement across the four factors was significantly different $(p=0.04)$.

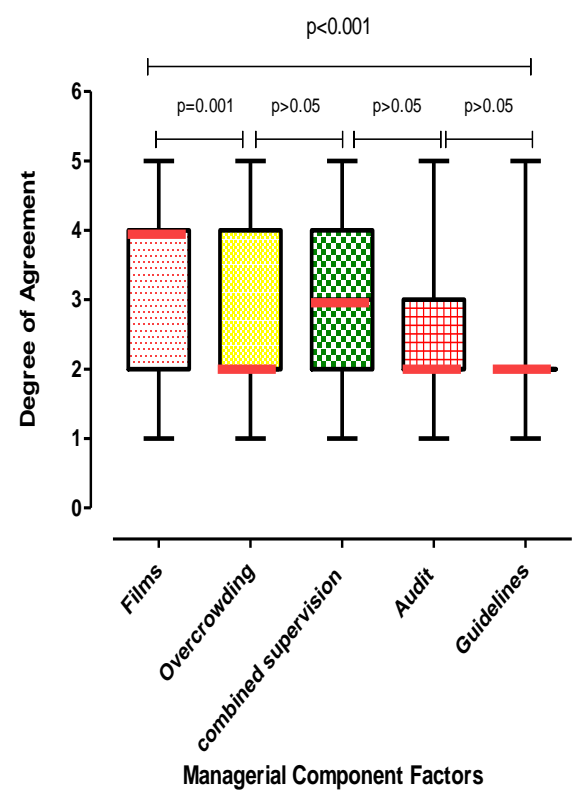

Figure 1A

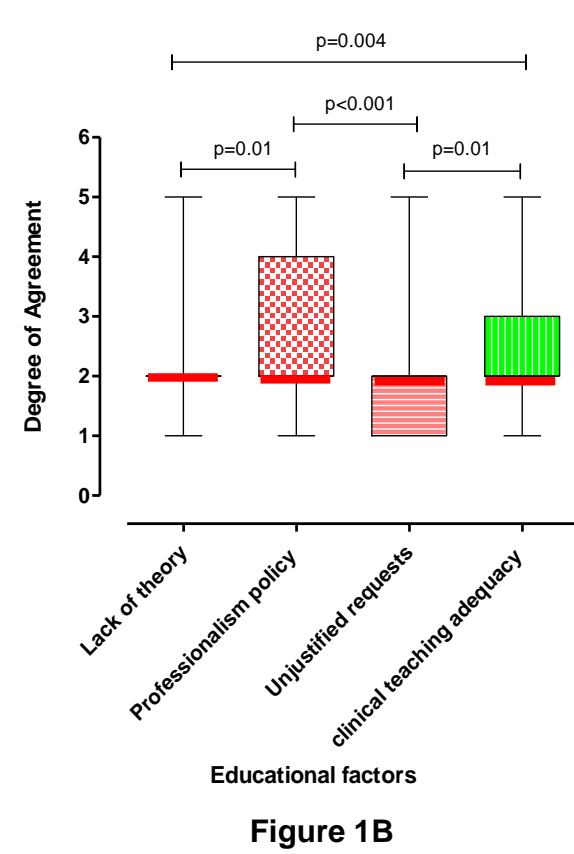

Figure 1B

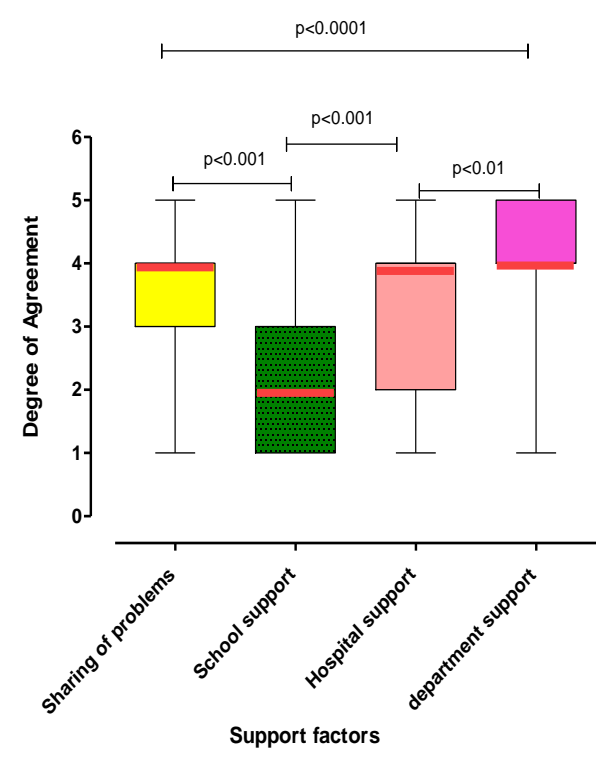

Figure 1C

Figure 1: Factors affecting clinical supervision 
Factors related to the supportive component of the clinical supervision of radiography students

As depicted in Figure 1C, most respondents $[\mathrm{N}=84(70 \%)]$ rated their agreement at 4 or 5 with the statement that radiography students share problems related to their learning with supervising radiographers. The academic institution support to supervising radiographers where $\mathrm{N}=81(67.5 \%)$ indicated that the support is inadequate, giving a median rating of 2 (IQR 1-3). The majority $[\mathrm{N}=71 \quad(59.1 \%)]$ of respondents rated the support they got from the hospital management as low, as seen by the rating of 3 or less. The support from the radiology department management was, however, rated as being very high with an agreement level of 4 or higher by the majority $[\mathrm{N}=97(80.9 \%)]$. There was a significant difference in the agreement levels $(p<0.0001)$ across the four factors except for the comparison between non-sharing of learning problems by radiography students $(p>0.05)$.

Comparison of aggregate evaluation with standard

The median rating for managerial was lower [56 (IQR 48-60)] and statistically different from the standard grading mark of 60 required for accreditation as a mid-level training institution $(p=0.001)$. The rating of the educational system was significantly less $(p<0.001)$ with the median at 45 (IQR 28-56). There was a significantly higher median rating of 66 (IQR 60-75) in the support category compared to the standard score of 60 ( $P<0.0001)$. Figure 2 shows the comparison rating across the categories. There was significant difference across the categories $\mathrm{p}<0.0001$.

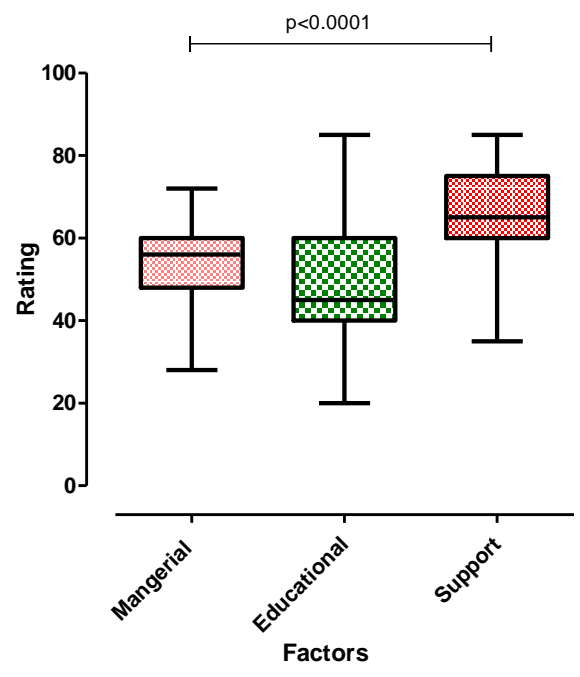

Figure 2: Comparison of factors against standard rating for accreditation

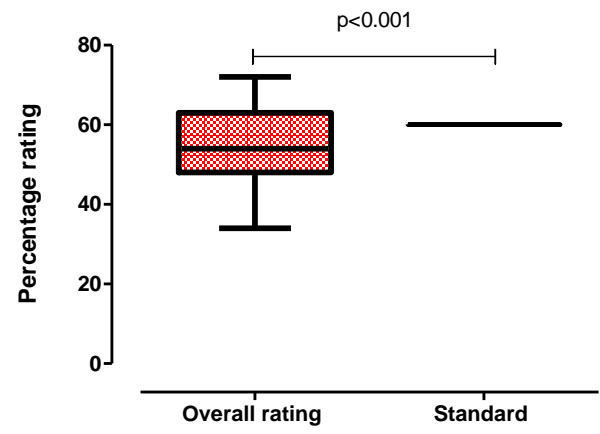

Figure 3: Rating of the current clinical supervision system 
The rating in the three categories (managerial, educational and supportive) were aggregated and an overall rating obtained. The aggregate median rating by the respondents was 54 (IQR 48-63). Using the Wilcoxon Signed Rank Test, with $60 \%$ as the hypothetical median, there was a significant difference $(p<0.001)$ noted (Figure 3). Most respondents $[\mathrm{N}=81(67.5 \%)]$ rated the current clinical supervising system for radiography students in Zambia as unsatisfactory.

\section{Discussion}

Clinical supervision of radiography students is an important aspect of radiography training. There are three components of clinical supervision: managerial, educational, and supportive (University of Ottawa, 2011). The managerial component deals with the management and organisation of training resources, the educational component involves the imparting of knowledge to students, and the supportive component deals with supporting students with learning difficulties and disabilities (Murphy, 2011; Baker \& Latham, 2012; Walsh, 2020).

Regarding managerial factors investigated, on average, the rating of the managerial factors was below $60 \%$ as per the TEVETA requirement. The finding concerning $X$-ray film availability is similar to those of Kyei et al., (2015) where the majority (76.2\%) of radiography students indicated adequate $\mathrm{X}$-ray films during clinical training. In the context of this study, an adequate supply of X-ray films is a facilitating factor in clinical supervision as it provides continuity in the radiology services and clinical training of radiography students (Bwanga \& Sichone, 2020). Inadequacy, in other factors such as equipment can also serve as a demotivator for supervising radiographers.

In our study, a lack of clinical supervision guidelines was noted as an inhibiting factor in clinical supervision. This finding was also affirmed by Bvumbwe et al., (2015) who conducted a study in Malawi and found that clinical supervisors of nursing students were frustrated due to a lack of guidelines. Harden and Laidlaw (2020) point out that guidelines inform the clinical supervisors about the contemporary clinical supervision practices to follow, approved by the training institutions.

The shortage of radiographers in Zambia was noted in the National Human Resources for Health Strategic Plan of 2011-2015 as a challenge in the delivery of quality radiology services (Ministry of Health, 2011). Hence the need to scale up training. However, this has resulted in overcrowding of clinical sites as noted by supervising radiographers in our study. This was found to be an inhibiting factor for the clinical supervision of radiography students because of a busy learning environment.

In a study conducted in the UK by Sutton (2013), supervising radiographers found it challenging to supervise first and third-year radiography students at the same time because of different objectives and levels of training. This was also seen to be a similar problem in our study, when supervising both diploma and degree radiography students in the same group. This was found to be an inhibiting factor to the facilitation of practice-based learning for radiography students.

The educational audit of clinical departments affiliated with the schools of radiography is part of the quality assurance programme (College of Radiographers, 2005). Such audits enable students to have appropriate educational opportunities, improve students' and supervising radiographers' experiences and identify areas that need to maintain, improve, and develop the quality of clinical departments (University of Nottingham, 2020). Unfortunately, most of the supervising radiographers in our study indicated a lack of educational audits in their respective departments. A survey also conducted in European countries by English et al., (2017) found most $(68.8 \%)$ of the radiography clinical departments had no regular educational audits. A lack of regular monitoring of learning environments is an inhibiting factor in clinical supervision.

The academic institutions have a responsibility to adequately cover theory as per curriculum 
before sending radiography students for clinical training (College of Radiographers, 2006). However, most of the supervising radiographers in our study indicated that radiography students lacked sufficient knowledge of theory. This can add workload to supervising radiographers in teaching theory before giving opportunities to students to undertake clinical practice. The inadequate coverage of theory is therefore an inhibiting factor to the clinical supervision of radiography students.

Supervising radiographers are responsible for teaching professional ethics, morals, and standards of behaviour for students. One of the teaching tools used is a professionalism policy statement that defines the characteristics of the expected behaviour of students during clinical training (Kirk, 2007). According to Kirk (2007), the professionalism policy statement should describe the processes relating to reporting channels of those students showing unprofessional behaviours, due process, absence of retaliation for clinical staff and peers who report unprofessional behaviours, remediation processes, and follow-up meetings between the supervising radiographer and student.

Requests for and performances of radiological examinations are guided by the radiation protection principle of justification. A radiological examination is justified if the benefits to the individual patient will do more good than harm (Bwanga \& Nyanga, 2020). In this study, most of the supervising radiographers indicated that medical practitioners sometimes request unjustified radiological examinations. A study conducted by Mung'omba and Botha (2017) also found medical doctors requesting unjustified examinations which negatively affected the delivery of radiology services. Our study has further identified how this issue affects the training of radiography students. Deviating from best practices by accepting unjustified examinations can negatively affect supervising radiographers in teaching professionalism to students. Acting unprofessionally can model bad practices for students (Walsh, 2020).
According to the College of Radiographers (2012), supervising radiographers should develop teaching and supervisory skills and act as a resource for radiography students seeking information and guidance. In our study, most of the supervising radiographers reported a lack of knowledge in the educational principles related to clinical supervision. Global literature also reports a lack of clinical teaching skills amongst supervising radiographers (English et al., 2017; Bwanga \& Sichone, 2020; Thompson \& Taylor, 2020). Walsh (2020) states that understanding how people learn can help supervisors to adjust their teaching appropriately and improve the effectiveness and efficiency of learning. To improve the delivery of clinical education, there is a need to develop a clinical supervision training programme for radiographers and the provision of continuous professional development (CPD) related to the facilitation of practice-based learning.

Supervising radiographers reported that most of the radiography students had positive attitudes towards sharing their learning difficulties. This finding agrees with what is reported in the literature. A study in the UK by Murphy (2011), found that all radiography students with learning disabilities had made a disclosure about it to the university and these had been communicated to respective clinical departments. This is a facilitating factor in the clinical supervision of students with learning difficulties as it can allow supervising radiographers to provide appropriate support and if possible, make reasonable adjustments to suit the student's needs (Murphy, 2011; Walsh, 2020).

The support from academic institutions is paramount in clinical education (College of Radiographers, 2012). However, most of the supervising radiographers in our study were dissatisfied with the support received from academic institutions. This finding agrees with other previous nursing studies that have identified a lack of support from the faculty as a hindrance to clinical supervision (Msiska et al., 2014; Bvumbwe et al, 2015; Setati \& Nkosi, 2017). According to Boss et al., (2015) a lack of support from faculty creates a feeling of abandonment amongst clinical supervisors. 
Although hospital management provided the clinical learning environment for radiography students, most of the supervising radiographers were dissatisfied with their support. This concurs with a nursing study conducted by Bos et al., (2015) where clinical supervisors stated that they did not receive support from their hospital management due to a lack of interest in the students' learning. Interestingly, most of the supervising radiographers in our study were satisfied with the support from the radiology department managements. Bvumbwe et al., (2015) state that clinical supervisors feel appreciated when departmental managers are involved in the training of students. This can create a conducive clinical learning environment for students.

\section{Conclusion}

Supervising radiographers rated the current system of clinical supervision of radiography students as being unsatisfactory. A closer relationship, with more collaborations amongst key stakeholders (schools of radiography, radiology departments and Radiological Society of Zambia [RSZ]) is necessary to overcome the inhibiting factors to clinical supervision identified in the study. Due to a lack of literature on this subject in radiography, there is a need for more research on the facilitation of practice-based learning for radiography students.

\section{Conflict of interest \\ None}

\section{References}

Baker, S.S., \& Latham, P. (2012). Introduction to clinical education, in Introduction to radiologic sciences and patient care, edited by Adler, A., \& Carlton, R. (5th edition). St. Louis: Elsevier Saunders. Pp43-50.

Bos, E., Silen, C., \& Kaila, P. (2015). Clinical supervision in primary health care: experiences of district nurses as clinical supervisors-a qualitative study. BMC Nursing, 14 (39): 1-8.

Bryman, A. (2016). Social research methods (5th edition). Oxford: University Press.

Bvumbwe, T., Malema, A., \& Chipeta, M. (2015). Registered nurses' experiences with clinical teaching environment in Malawi. Open Journal of Nursing, 5: 927-934.

Bwanga, O., \& Nyanga, I. (2020). Justification of imaging medical exposures: a review of literature to guide evidence-based practice in Zambia. Medical Journal of Zambia, 47(4): 318 326.

Bwanga, O., \& Sichone, M.J. (2020). Experiences of clinical supervisors regarding the clinical training of radiography students in Zambia. The South African Radiographer, 58(2): 22-28.

College of Radiographers (2005). Guidance on approval and accreditation of practice placements at all levels of pre-registration education. London: The College of Radiographers.

College of Radiographers (2006). Guidance and strategies for effective relationships between education providers, placement providers and learners. London: The College of Radiographers.

College of Radiographers (2012). Quality standards for practice placements. London: The College of Radiographers.

England, A., Gemeren, S.G., Henner, A., Kukkes, T., Pronk-Larive, D., Rainford, L., et al., (2017). Clinical radiography education across Europe. Radiography, 23 (1): 7-15.

Harden, R.M., \& Laidlaw, J.M. (2020). Essential skills for a medical teacher-An introduction to teaching and learning in medicine ( $3^{\text {rd }}$ edition). London: Elsevier.

Health Professions Council of Zambia (2014). Professional code of ethics and discipline: fitness to practice. Lusaka: HPCZ.

Kyei, K.A., Antwi, W.K., Bamfo-Quaicoe, K., \& Offei, R.O. (2015). Challenges faced by radiography students during clinical practice. Clinical Medicine Research, 4 (3-1): 36-41.

Kirk, L. (2007). Professionalism in medicine: definitions and considerations for teaching. Baylir University Medical Center Proceedings, 20 (1): 13-16.

Lusaka Apex Medical University (2017). Bachelor of science in diagnostic radiography curriculum. Lusaka: LAMU.

Ministry of Health. National Health Strategic Plan 2011-2015 (2011). Lusaka: Ministry of Health.

Msiska, G., Smith, P., \& Fawcett, T. (2014). The "lifeworld" of Malawian undergraduate student nurses: The challenge of learning in resource poor clinical settings. International Journal of Africa Nursing Sciences, 1: 35-42. 
Murphy, F. (2011). On being dyslexic: Student radiographers' perspectives. Radiography, 17 (2): 132-138.

Mung'omba, B., \& Botha, A.D.H. (2017). Core competencies of radiographers working in rural hospitals of KwaZulu-Natal, South Africa. African Journal of Primary Health Care and family Medicine, 9 (1): 2071-2928.

Radiological Society of Zambia (2018). Code of conduct for radiographers. Lusaka: Radiological Society of Zambia.

Setati, C.M., \& Nkosi, Z.Z. (2017). The perceptions of professional nurses on student mentorship in clinical areas: A study in Polokwane municipality hospitals, Limpopo province. Health SA Gesondheid, 22: 130-137.

Sutton, R. (2012). A focused ethnography of radiotherapy students' learning on their first clinical placement. Doctorate thesis. Cardiff University. Cardiff.

Technical Education Vocational and Entrepreneurship Training Authority (2012). Standard for registration TEVETA institutions. Lusaka: TEVETA.
Technical Education Vocational and Entrepreneurship Training Authority (2018). Diploma in radiography. Lusaka: TEVETA.

Thomas. A.M.K., \& Banerjee, A.K. (2013). The history of radiology. Oxford: Oxford Medical Histories.

Thompson, A., \& Taylor, D. (2020). Finding ways to support radiographers as teachers. Journal of Medical Radiation Sciences, 67(3):199-207.

University of Nottingham (2020). Educational audit of practice placements. Available from https://www.nottingham.ac.uk/healthsciences/pr actice/nursing/audit/index.aspx (accessed 20 June 2021)

University of Ottawa (2011). The clinical supervisor's functions. Available from http://health.uottawa.ca/pdf/3principedebase-en. pdf (accessed 10 May 2021).

University of Zambia (2017). Bachelor of science in diagnostic radiography curriculum. Lusaka: UNZA.

Walsh, D. (2020). The nurse mentor's handbooksupporting students in clinical practice (3rd edition). Berkshire: Open University Press. 